\title{
Chiral three-body force and monopole properties of shell-model Hamiltonian
}

\author{
Tokuro Fukui ${ }^{1, *}$, Luigi Coraggio ${ }^{1}$, Luca De Angelis ${ }^{1}$, Angela Gargano ${ }^{1}$, Nunzio Itaco $^{1,2}$, Yuanzhuo $\mathrm{Ma}^{3}$, and Furong Xu ${ }^{3}$ \\ ${ }^{1}$ Istituto Nazionale di Fisica Nucleare, Sezione di Napoli, Complesso Universitario di Monte S. Angelo, Via Cintia I-80126 Napoli, \\ Italy \\ ${ }^{2}$ Dipartimento di Matematica e Fisica, Università degli Studi della Campania “Luigi Vanvitelli”, viale Abramo Lincoln 5 - I-81100 \\ Caserta, Italy \\ ${ }^{3}$ School of Physics, and State Key Laboratory of Nuclear Physics and Technology, Peking University, Beijing 100871, China
}

\begin{abstract}
So far, the nature of three-nucleon forces (3NFs) derived by the chiral effective field theory has been intensively investigated by various theoretical approaches. In this work, to address the chiral 3NF within the shell-model framework, three-body matrix elements are formulated in terms of the harmonic oscillator basis functions, by adopting the nonlocal regulator. We perform a benchmark test for $p$-shell nuclei in order to confirm our framework. Then we show that the contribution of the $3 \mathrm{NF}$ to the monopole component of the effective shell model Hamiltonian plays an essential role to account for the shell evolution of $f p$-shell nuclei.
\end{abstract}

\section{Introduction}

The realistic shell model (RSM) employing a realistic nuclear force is one of the theoretical tools describing the nuclear structure microscopically and exploring the nature of the nuclear force. As regards obtaining the state-of-theart nuclear force, the chiral effective field theory [1] has a great advantage that many-body forces appear on an equal footing.

Zuker suggested by a phenomenological way that the RSM Hamiltonian consisting of a realistic two-nucleon force (2NF) needs to be corrected due to the absence of three-nucleon forces (3NFs) [2]. In particular, the correction to its monopole component, which is responsible for the evolution of the spherical mean field, was found to be crucial. Indeed, it was shown that, by the RSM calculations, $3 \mathrm{NFs}$ play a significant role to explain the neutron drip line of the oxygen isotope [3]. The effect of the chiral $3 \mathrm{NF}$ on the Ca isotopes was also investigated [4], but how its monopole correction is important in this region remains unclear.

We aim to clarify the $3 \mathrm{NF}$ contribution to the monopole Hamiltonian for $f p$-shell nuclei including the $\mathrm{Ca}$ isotopes. To this end, we derive new formalism of the three-body matrix element and perform a benchmark test for $p$-shell nuclei.

\section{Theoretical Framework}

Within the RSM framework, we separate the Hilbert space into two parts, the valence-nucleon and inert-core parts, and explicitly address the valence nucleons only. First, we need to compute the three-body matrix element with the harmonic oscillator $(\mathrm{HO})$ basis functions as follows:

\footnotetext{
*e-mail: fukui@na.infn.it
}

(1) Transformation from the three-body $J T$-coupled HO states into the Jacobi-HO states [5], by Talmi transformation [6-11], with the separation of the center-of-mass and relative motions.

(2)Antisymmetrization of the Jacobi-HO states by diagonalizing the three-body antisymmetrizer $[12,13]$.

(3)Evaluation of the three-body matrix element regularized by the nonlocal regulator with the Jacobi-HO basis at the chiral next-to-next-to-leading order $\left(\mathrm{N}^{2} \mathrm{LO}\right)$ [14].

The steps (1) and (2) are essentially same as those reported in Ref. [15], whereas the step (3) is newly developed by the present work [16]. Next, the three-body operator appearing in the Hamiltonian is reduced to the one- and two-body operators by the normal-ordering approximation [17]. Then, in order to incorporate the chiral two-body interaction at next-to-next-to-next-to-leading order [14] and the normal-ordered three-body interaction into the RSM, we adopt the many-body perturbation theory [18], and thus obtain the effective Hamiltonian associated with the valence-nucleon space.

The further description of our theoretical framework is reported in Refs. [16, 19], as well as the model spaces and parameters adopted for the calculations.

\section{Results}

\subsection{Benchmark test for $p$-shell nuclei}

Figure 1 shows the energy spectra relative to the ground state (g.s.) for the selected $p$-shell nuclei, ${ }^{6} \mathrm{Li}$ and ${ }^{10} \mathrm{~B}$. Our results obtained by the RSM are compared with the experimental data (Exp) [20] and those by the ab initio no-core shell model (NCSM) [21, 22]. One finds from Figs. 1(a) and (c) that, even with the 2NF only, the RSM gives the 

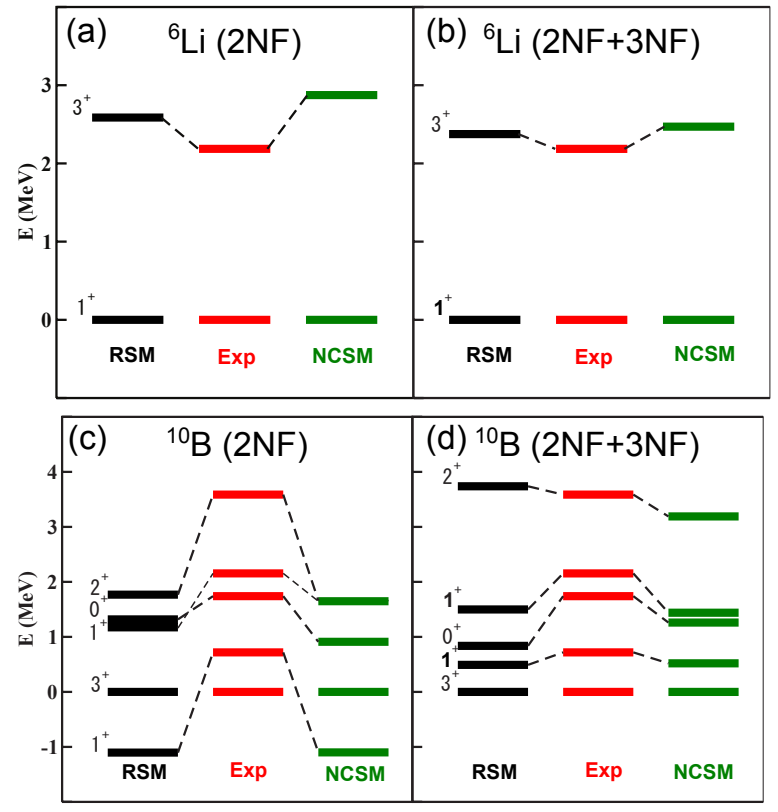

Figure 1. The comparison of the low-lying energy spectra of ${ }^{6} \mathrm{Li}$ and ${ }^{10} \mathrm{~B}$ computed by the RSM and NCSM, together with the experimental data. See text for details.

low-lying spectra comparable to those by the NCSM, although they are not consistent with the data. Indeed, both models at the $2 \mathrm{NF}$ level cannot provide the correct g.s. for ${ }^{10} \mathrm{~B}$. Then, as shown in Figs. 1(b) and (d), the inclusion of the $3 \mathrm{NF}$ plays a significant role on the spectra and improves the levels consistent with the data, with the correct g.s. of ${ }^{10} \mathrm{~B}$. The similar results are obtained also for ${ }^{8} \mathrm{Li}$, ${ }^{8} \mathrm{Be},{ }^{8} \mathrm{~B},{ }^{11} \mathrm{~B},{ }^{12} \mathrm{C}$, and ${ }^{13} \mathrm{C}[16]$.

In Fig. 2(a), the consistency between the RSM and NCSM at the 2NF level is also confirmed from the g.s. energy $E_{\text {g.s. }}$ of the $p$-shell nuclei as a function of the mass number $A$. We select the systems with the neutron number equal to the proton number. The $2 \mathrm{NF}$ only is not enough to gain $E_{\text {g.s. }}$ consistent with the experimental data. The NCSM with the $3 \mathrm{NF}$ significantly improves the results, which coincide with the data, while the RSM with the $3 \mathrm{NF}$ remains overestimate the data, represented in Fig. 2(b). This may suggest that the normal-ordering approximation

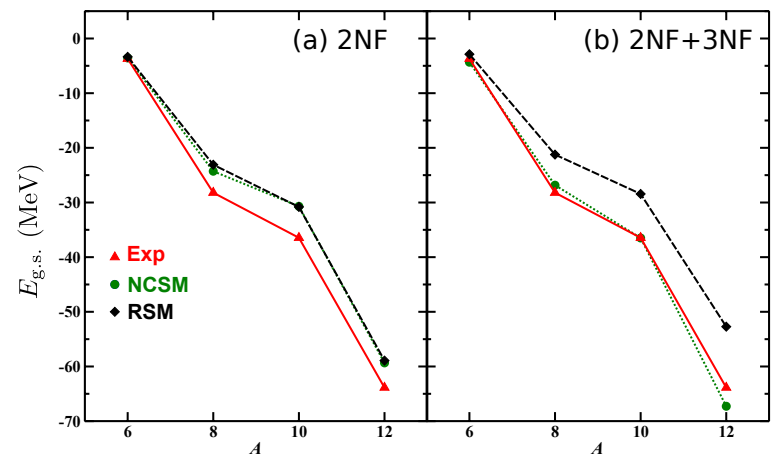

Figure 2. The g.s. energy for the selected $p$-shell nuclei obtained with (a) the 2NF only and (b) 2NF plus 3NF in both models.

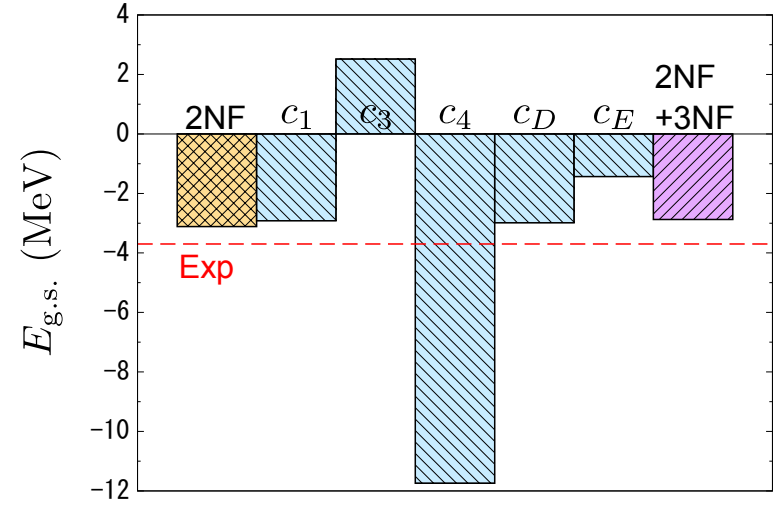

Figure 3. The individual contribution of each term of the $3 \mathrm{NF}$ to $E_{\text {g.s. }}$ of ${ }^{6} \mathrm{Li}$. See text for details.

is not sufficient for $E_{\text {g.s. }}$, although it works for the relative energy spectra. As mentioned above, we adopt the normalordering approximation for the three-body operator in the RSM, whereas the NCSM explicitly addresses it without the approximation. It is required to make the approximation more precise.

The chiral $3 \mathrm{NF}$ at $\mathrm{N}^{2} \mathrm{LO}$ can be decomposed into five terms associated with each low-energy constant (LEC). The two-pion exchange term involves the three LECs, $c_{1}$, $c_{3}$, and $c_{4}$, while the one-pion exchange plus contact term (the contact term) has the LEC $c_{D}\left(c_{E}\right)$. Figure 3 expresses the individual contribution of each of those terms to $E_{\text {g.s. }}$ of ${ }^{6} \mathrm{Li}$. The experimental value is shown by the dashed line and the calculated result only with the $2 \mathrm{NF}$ is given by the cross-hatched bar. We see that the $c_{4}$ term (declininghatched bar) has the largest contribution to $E_{\mathrm{g} . \mathrm{s} \text {. }}$. This is consistent with the fact that the two-pion exchange term is the most dominant component in the chiral $3 \mathrm{NF}$ at $\mathrm{N}^{2} \mathrm{LO}$ (see, for example, Ref. [23]). It is also clearly exhibited that there is a large coherent effect when all the terms exist, since an incoherent sum of each $c_{i}$ contribution, which is always attractive except for the $c_{3}$ one, does not reproduce the full result (soaring-hatched bar).

\subsection{Shell evolution on $f p$-shell nuclei}

In the RSM calculations for the $f p$-shell nuclei, we adopt the single-particle energy corrected by the 3NF [19], and

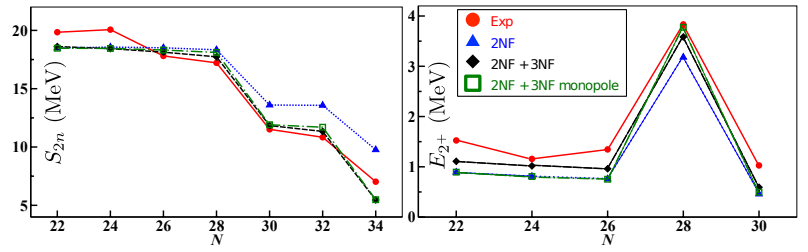

Figure 4. The comparison of the measured data [20] and RSM results on the two-neutron separation energy $S_{2 n}$ and the $2^{+}$excitation energy $E_{2^{+}}$for the Ca isotopes. Three types of the RSM calculations are performed: the $2 \mathrm{NF}$ only (triangle), $2 \mathrm{NF}$ plus $3 \mathrm{NF}$ (filled square), and $2 \mathrm{NF}$ with the monopole correction by the $3 \mathrm{NF}$ (open square). 


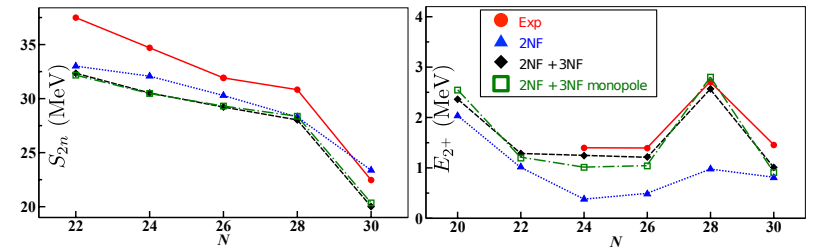

Figure 5. Same as Fig. 4 but for the Ni isotopes.

thus, in the following we discuss the $3 \mathrm{NF}$ effect on the two-body term. Figure 4 shows the two-neutron separation energy $S_{2 n}$ and the $2^{+}$excitation energy $E_{2^{+}}$as a function of the neutron number $N$ for the Ca isotopes. One sees that experimental $S_{2 n}$ (circle) suddenly drops from $N=28$ to 30 owing to filling the neutron $0 f_{7 / 2}$ orbit. The RSM performed with the $2 \mathrm{NF}$ plus $3 \mathrm{NF}$ (filled square) satisfactory simulates this behavior, as well as the $2 \mathrm{NF}$ calculation with the monopole correction by the 3NF (open square), in contrast to the calculation only with $2 \mathrm{NF}$ (triangle) failing to explain this sudden drop. This clearly presents that the $3 \mathrm{NF}$ plays an important role to be responsible for the shell evolution on the $\mathrm{Ca}$ isotopes, and the $3 \mathrm{NF}$ contribution to the monopole Hamiltonian is essential. For $E_{2^{+}}$, all the calculations give the similar results, which are slightly underestimated compared to the measured data. However, the experimental $E_{2^{+}}$at $N=28$ coincides well with the RSM results by the $3 \mathrm{NF}$ and $2 \mathrm{NF}$ with the monopole correction, while the result of the $2 \mathrm{NF}$ only remains lower than the data. Thus we find that the $3 \mathrm{NF}$ is substantial to depict the closure property.

We show in Fig. 5 the comparison of the RSM results with the measured data for $S_{2 n}$ and $E_{2^{+}}$of the Ni isotopes. The RSM globally underestimates the measured $S_{2 n}$ but the 3NF result well reproduces the drop of $S_{2 n}$ at $N=30$, the result of the 2NF with the monopole correction as well. In contrast, the $2 \mathrm{NF}$ calculation cannot describe this feature. The above consequence obtained from $S_{2 n}$ is qualitatively consistent with that for the Ca isotopes. For $E_{2^{+}}$, however, we obtain results very different from the Ca case. The RSM only with the 2NF gives smaller values of $E_{2^{+}}$, specifically at $N=28$, compared to the other two calculations, which provide $E_{2^{+}}$consistent with the measured data. Thus we find the $3 \mathrm{NF}$ responsible for the closure property of the $\mathrm{Ni}$ isotopes is more prominent than that of the $\mathrm{Ca}$ case. This can be interpreted by scrutinizing the effective single-particle energy (ESPE).

The ESPE is the evolved single-particle energy due to the monopole interaction, which is the angularmomentum averaged two-body interaction (see, for instance, Ref. [24]). Using the ESPE, we are able to elucidate how the single-particle structure evolves as the number of neutrons increases.

Figure 6 displays the neutron ESPEs relevant for the $\mathrm{Ca}$ isotopes as a function of $N$. The distance between each ESPE is almost constant as $N$ increases, and they are well separated from each other. In particular, we see the large gap between the $0 f_{7 / 2}$ and $1 p_{3 / 2}$ ESPEs, which accounts for the closure property at $N=28$. The inclusion of the

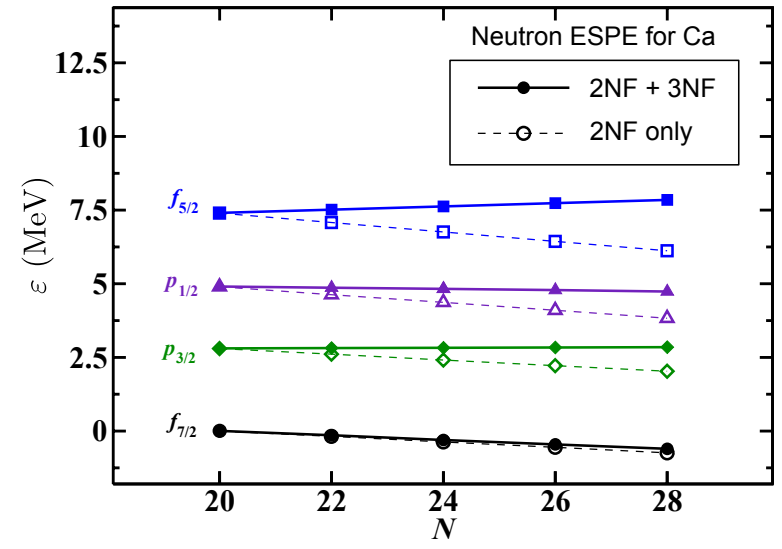

Figure 6. The neutron ESPEs calculated with the 2NF plus $3 \mathrm{NF}$ (filled symbols) and the $2 \mathrm{NF}$ only (open symbols), for the $\mathrm{Ca}$ isotopes as a function of $N$.

3NF enlarges this gap by about $0.7 \mathrm{MeV}$ at $N=28$, although each ESPS remains isolated from the others. This induces the more substantial shell closure.

The neutron and proton ESPEs of the Ni isotopes as a function of $N$ are presented in Fig. 7. We find that the neutron $0 f_{7 / 2}$ ESPE is well separated from the other three ESPEs. This makes the shell closure stronger on ${ }^{56} \mathrm{Ni}$. In contrast, the grouping of the neutron ESPEs of the $1 p_{3 / 2}$, $1 p_{1 / 2}$, and $0 f_{5 / 2}$ orbits enhances the collectivity reducing the closure. The similar feature is observed also in the proton ESPEs. Consequently, due to the interplay of these op-

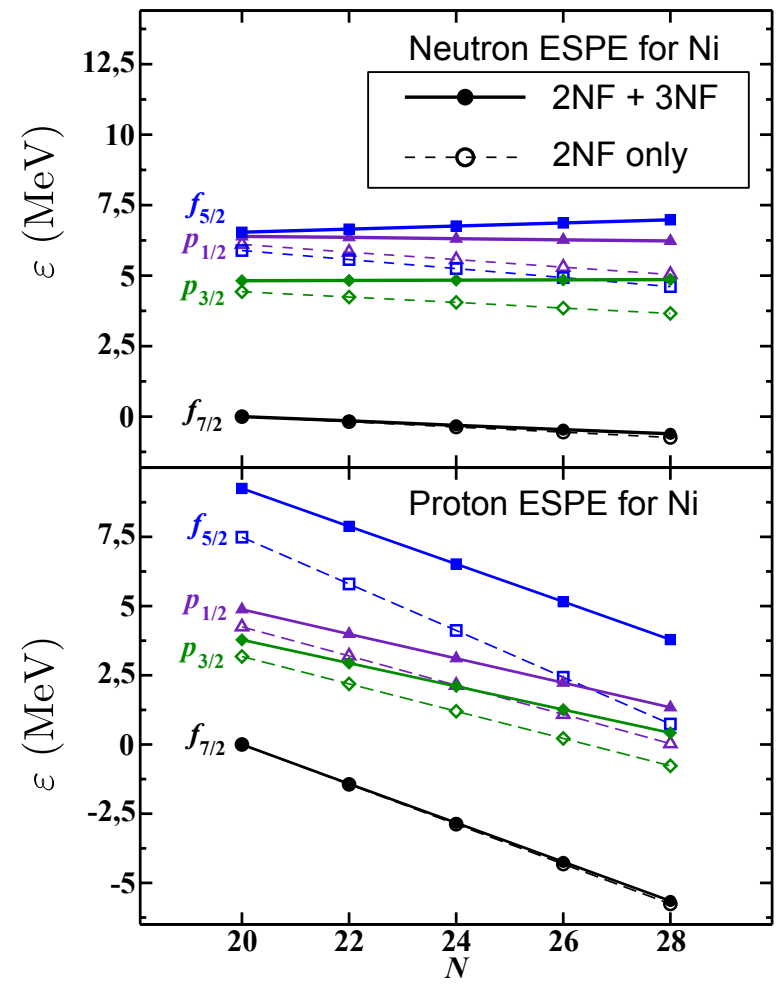

Figure 7. The neutron and proton ESPEs calculated with the $2 \mathrm{NF}$ plus $3 \mathrm{NF}$ (filled symbols) and the 2NF only (open symbols), for the Ni isotopes as a function of $N$. 
posite effects, the shell closure on ${ }^{56} \mathrm{Ni}$ is slightly smaller than that on ${ }^{48} \mathrm{Ca}$, evidenced by the value of $E_{2^{+}}$of ${ }^{56} \mathrm{Ni}$ that is about $1 \mathrm{MeV}$ smaller than that of ${ }^{48} \mathrm{Ca}$.

When we include the $3 \mathrm{NF}$, the gap between the $0 f_{7 / 2}$ and $1 p_{3 / 2}$ orbits increases by about $1 \mathrm{MeV}$ in both neutron and proton ESPEs. Moreover, the $0 f_{5 / 2}$ orbit of the proton ESPE is strongly enhanced by the $3 \mathrm{NF}$ at $N=28$. Therefore, the $3 \mathrm{NF}$ effect observed on $E_{2^{+}}$of the $\mathrm{Ni}$ isotopes is more distinguished than that of the $\mathrm{Ca}$ case.

This fact is also understood by the property of the twopion exchange contributions. As presented in Fig. 3 as an example, the $3 \mathrm{NF}$ is generally dominated by the $c_{4}$ term. However, due to the charge conservation, the $c_{4}$ term vanishes when the three particles are identical. Naively, such situation in the $\mathrm{Ca}$ isotopes is relatively important than that in the Ni case, and thus we can explain the different behavior of the $3 \mathrm{NF}$ on the $\mathrm{Ca}$ and $\mathrm{Ni}$ isotopes.

\section{Perspectives}

We require a prescription beyond the normal-ordering approximation, which is equivalent to the first-order term in the perturbative expansion of the interaction. The inclusion of the second-order terms in addition to the normalordering approximation would modify the single-particle energies. We expect, therefore, that the second-order contribution meliorates the simulation of $E_{\text {g.s. }}$, as well as $S_{2 n}$ and $E_{2^{+}}$. In order to systematically investigate the contribution of the $3 \mathrm{NF}$, we plan to apply the present framework to heavier systems, such like candidates of the neutrinoless double-beta decay. The implementation of the chiral $3 \mathrm{NF}$ into the Gamow shell model [25-29] is also interesting since both $3 \mathrm{NF}$ and continuum effects are expected to be crucial for neutron-rich nuclei near drip lines. Indeed, the oxygen drip line is explained by a repulsive property of the $3 \mathrm{NF}$ [3], while the continuum degrees of freedom act as a attractive nature [30]. The interplay of the $3 \mathrm{NF}$ and the continuum effects needs to be clarified.

\section{References}

[1] S. Weinberg, Phys. A 96, 327 (1979)

[2] A.P. Zuker, Phys. Rev. Lett. 90, 042502 (2003)

[3] T. Otsuka, T. Suzuki, J.D. Holt, A. Schwenk, Y. Akaishi, Phys. Rev. Lett. 105, 032501 (2010)

[4] J.D. Holt, J. Menéndez, J. Simonis, A. Schwenk, Phys. Rev. C 90, 024312 (2014)

[5] R. Roth, A. Calci, J. Langhammer, S. Binder, Phys. Rev. C 90, 024325 (2014)

[6] I. Talmi, Helv. Phys. Acta 25, 185 (1952)
[7] T.A. Brody, M. Moshinsky, Tables of Transformation Brackets (Monografias del Instituto de Fisica, Mexico, 1960)

[8] M. Moshinsky, T.A. Brody, Rev. Mex. Fís. 9, 181 (1960)

[9] L. Trlifaj, Phys. Rev. C 5, 1534 (1972)

[10] B. Buck, A.C. Merchant, Nucl. Phys. A 600, 387 (1996)

[11] G.P. Kamuntavičius, R.K. Kalinauskas, B.R. Barrett, S. Mickevičius, D. Germanas, Nucl. Phys. A 695, 191 (2001)

[12] P. Navrátil, B.R. Barrett, W. Glöckle, Phys. Rev. C 59, 611 (1999)

[13] P. Navrátil, G.P. Kamuntavičius, B.R. Barrett, Phys. Rev. C 61, 044001 (2000)

[14] R. Machleidt, D. Entem, Phys. Rep. 503, 1 (2011)

[15] P. Navrátil, Few-Body Syst. 41, 117 (2007)

[16] T. Fukui, L. De Angelis, Y.Z. Ma, L. Coraggio, A. Gargano, N. Itaco, F.R. Xu, Phys. Rev. C 98, 044305 (2018)

[17] R. Roth, S. Binder, K. Vobig, A. Calci, J. Langhammer, P. Navrátil, Phys. Rev. Lett. 109, 052501 (2012)

[18] L. Coraggio, A. Covello, A. Gargano, N. Itaco, T. Kuo, Ann. Phys. 327, 2125 (2012)

[19] Y.Z. Ma, L. Coraggio, L. De Angelis, T. Fukui, A. Gargano, N. Itaco, F.R. Xu, Phys. Rev. C 100, 034324 (2019)

[20] Data extracted by using the nndc on-line data service from the ensdf database, file revised as of march 15, 2019, https: //www.nndc. bnl.gov/ensdf

[21] P. Navrátil, V.G. Gueorguiev, J.P. Vary, W.E. Ormand, A. Nogga, Phys. Rev. Lett. 99, 042501 (2007)

[22] P. Maris, J.P. Vary, P. Navrátil, Phys. Rev. C 87, 014327 (2013)

[23] H. Krebs, A.M. Gasparyan, E. Epelbaum, Phys. Rev. C 98, 014003 (2018)

[24] A. Umeya, K. Muto, Phys. Rev. C 74, 034330 (2006)

[25] R. Liotta, E. Maglione, N. Sandulescu, T. Vertse, Physics Letters B 367, 1 (1996)

[26] N. Michel, W. Nazarewicz, M. Płoszajczak, K. Bennaceur, Phys. Rev. Lett. 89, 042502 (2002)

[27] R. Id Betan, R.J. Liotta, N. Sandulescu, T. Vertse, Phys. Rev. C 67, 014322 (2003)

[28] N. Michel, W. Nazarewicz, M. Płoszajczak, J. Okołowicz, Phys. Rev. C 67, 054311 (2003)

[29] R. Id Betan, R.J. Liotta, N. Sandulescu, T. Vertse, Phys. Rev. Lett. 89, 042501 (2002)

[30] K. Fossez, J. Rotureau, N. Michel, W. Nazarewicz, Phys. Rev. C 96, 024308 (2017) 